\title{
Barium-functionalized multiwalled carbon nanotube yarns as low-work-function thermionic cathodes
}

\author{
Lin Xiao, ${ }^{1, a)}$ Peng Liu, ${ }^{1}$ Liang Liu, ${ }^{1, b)}$ Kaili Jiang, ${ }^{1, c)}$ Xiaofeng Feng, ${ }^{1}$ Yang Wei, ${ }^{1}$ Li Qian, ${ }^{1}$ \\ Shoushan Fan, ${ }^{1}$ and Taihua Zhang ${ }^{2}$ \\ ${ }^{1}$ Department of Physics and Tsinghua-Foxconn Nanotechnology Research Center, Tsinghua University, \\ Beijing 100084, People's Republic of China \\ ${ }^{2}$ LNM, Institute of Mechanics, Chinese Academy of Sciences, Beijing 100080, People's Republic of China
}

(Received 14 November 2007; accepted 23 March 2008; published online 15 April 2008)

\begin{abstract}
Barium-functionalized multiwalled carbon nanotube yarns were fabricated by drawing and twisting multiwalled carbon nanotube forests through a solution containing barium nitrate. After heat activation under vacuum, the functionalized yarns were enriched in barium oxide due to the high surface-to-volume ratio of the nanotubes. The cathodes exhibited good thermionic properties, with a work function as low as $1.73-2.06 \mathrm{eV}$ and thermionic current density that exceeded $185 \mathrm{~mA} / \mathrm{cm}^{2}$ in a field of $850 \mathrm{~V} / 5 \mathrm{~mm}$ at $1317 \mathrm{~K}$. The barium-functionalized yarns had high tensile strength of up to $420 \mathrm{MPa}$ and retained strength of $\sim 250 \mathrm{MPa}$ after a $2 \mathrm{~h}$ activation process. (C) 2008 American Institute of Physics. [DOI: 10.1063/1.2909593]
\end{abstract}

Carbon nanotubes (CNTs) have great potential for use as field and thermionic emitters due to their excellent electrical and mechanical characteristics. Effective thermionic emission was obtained from micrometer-long defective CNTs, highlighting the possibility of using CNTs as miniature electron sources for e-beam and x-ray instruments and display applications. ${ }^{1}$ CNT yarns have recently attracted great interest due to their high electrical conduction, flexibility, and favorable mechanical properties. ${ }^{2-4}$ As electron sources, they have been demonstrated to exhibit large current-carrying capacity as field emitters ${ }^{5}$ with current density exceeding $100 \mathrm{~A} / \mathrm{cm}^{2}$ and thermionic emitters with large effective surface area and work function of $4.54-4.64 \mathrm{eV}$. ${ }^{6}$ We report here an effective method to produce barium-functionalized multiwalled CNT (Ba-MWCNT) yarns as low-work-function thermionic cathodes. The work function measured by the Richardson method was $1.73-2.06 \mathrm{eV}$ and the thermionic emission current density exceeded $185 \mathrm{~mA} / \mathrm{cm}^{2}$ for a field of $850 \mathrm{~V} / 5 \mathrm{~mm}$ at $1317 \mathrm{~K}$. The tensile strengths of the Ba-MWCNT yarn were $\sim 420$ and $250 \mathrm{MPa}$ before and after vacuum heating activation, respectively. These low-workfunction MWCNT yarns are suitable as thermal electron sources in devices such as vacuum fluorescent displays (VFDs), x-ray tubes, and electron guns.

MWCNT yarns were drawn from MWCNT forests synthesized by low-pressure chemical vapor deposition. ${ }^{4}$ The yarns then were drawn through droplets of solution containing a barium salt and simultaneously twisted. The solution was prepared by dissolving $9.2 \mathrm{~g}$ of barium nitrate, $7.4 \mathrm{~g}$ of strontium nitrate, and $0.3 \mathrm{~g}$ of calcium nitrate in $100 \mathrm{ml}$ of equal volumes of de-ionized water and ethanol. After evaporating the solvent at $100{ }^{\circ} \mathrm{C}$ in air, the Ba-MWCNT yarn was heated at $1100-1200 \mathrm{~K}$ for several minutes in vacuum by using a dc to decompose the nitrates to oxides. Scanning electron microscopy (SEM) images of an as-prepared yarn are shown in Figs. 1(a) and 1(b). The energy-dispersive x-ray spectrum (EDS) in Fig. 1(c) reveals the presence of barium

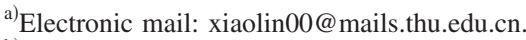

${ }^{b)}$ Electronic mail: liuliang @tsinghua.edu.cn.

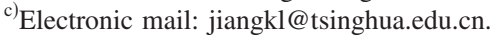

and strontium, but the calcium content was too low to be detected. The detection of oxygen but no nitrogen suggests that the nitrates had fully decomposed on the as-heated yarns.

The mass ratio of nitrates in the Ba-MWCNT yarns was determined (STA-499C thermal analyzer, Netzsch) before vacuum decomposition. Thermal gravimetric analysis was performed by increasing the temperature from 50 to $1200{ }^{\circ} \mathrm{C}$ at $10{ }^{\circ} \mathrm{C} / \mathrm{min}$ in oxygen. The mass percentage of nitrates was as high as $72 \%$ in the functionalized

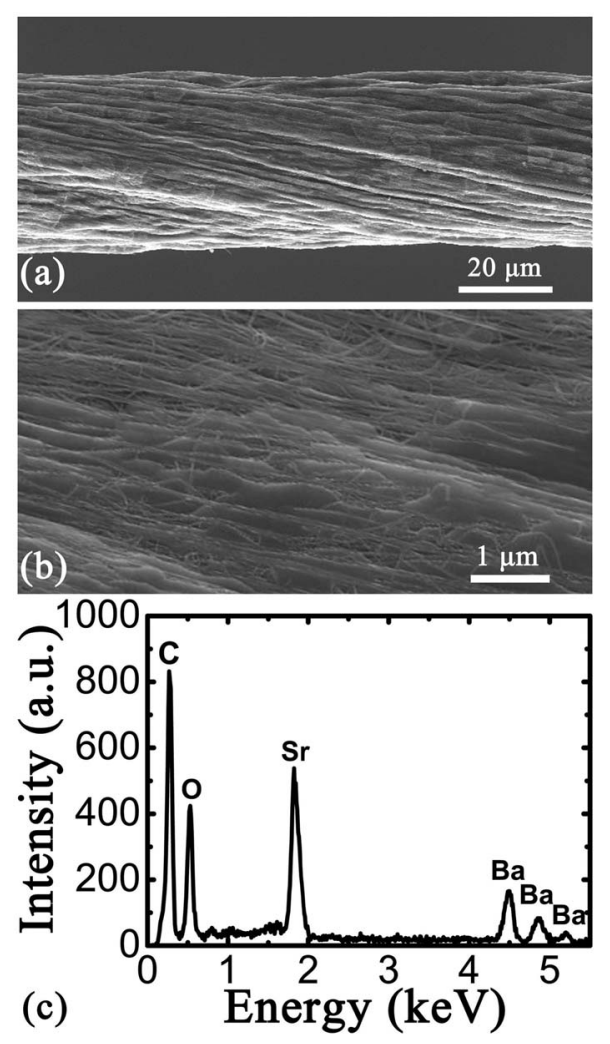

FIG. 1. SEM and EDS images of Ba-MWCNT yarn after heat treatment at $1127 \mathrm{~K}$ for $5 \mathrm{~min}$ in vacuum. [(a) and (b)] SEM images of the yarn at different magnifications. (c) EDS image of the yarn. 


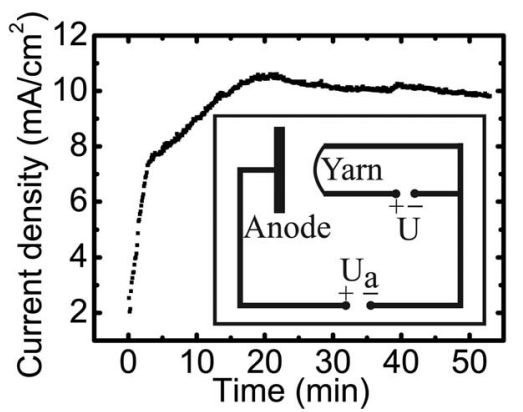

FIG. 2. Activation process for Ba-MWCNT yarn at $\sim 1150 \mathrm{~K}$ and $10^{-5} \mathrm{~Pa}$. Inset: Schematic diagram of the electric circuit used for thermionic emission tests.

yarns; SEM images revealed that most of them filled the spaces between nanotubes in the yarn. This high Ba loading means that Ba-MWCNT yarns are good storage thermionic cathodes.

The thermionic emission of Ba-MWCNT yarns was measured under vacuum $\left(10^{-5} \mathrm{~Pa}\right)$ by using the electric circuit shown in Fig. 2 (inset). A 2-cm-long Ba-MWCNT yarn with a diameter of $42 \mu \mathrm{m}$ used as the cathode (resistance $\sim 167 \Omega$ ) was heated by a dc power supply. The anode was a stainless steel plate placed $5 \mathrm{~mm}$ from the yarn. A Keithley 237 source meter was used to apply potential $U_{a}$ to the anode and test the emission current $I_{a}$ simultaneously. The current density $J_{a}$ was calculated as the emission current divided by the yarn's geometric area $\left(0.0264 \mathrm{~cm}^{2}\right)$. The temperature was derived by fitting the candescent light spectrum (KonicaMinolta CS-1000A spectroradiometer) of the yarn to that of black body radiation.

After nitrates on the MWCNT yarn decomposed to oxides, further heat treatment was carried out to produce a constant high emission current. This activation was necessary to produce an adequate level of $\mathrm{Ba}$ and to remove residual gases from the cathode. ${ }^{7}$ Figure 2 shows the current density for thermionic emission as a function of time at $\sim 1150 \mathrm{~K}$. The emission current rapidly increased during activation and reached a relatively steady state after $20 \mathrm{~min}$. The work function of the Ba-MWCNT yarn was tested after complete activation. Figure 3(a) shows the emission current density as a function of the anode voltage at different temperatures. A current density of $187 \mathrm{~mA} / \mathrm{cm}^{2}$ (total current $4.94 \mathrm{~mA}$, heating power $0.0602 \mathrm{~W}$ ) was obtained in an electric field of $850 \mathrm{~V} / 5 \mathrm{~mm}$ at $1317 \mathrm{~K}$. Attributed to the high barium oxide coverage on MWCNT yarn and the large surface area of CNTs, the current density could be further increased according to recent works. ${ }^{8,9}$

We recently used the Richardson method to determine the thermionic emission constant and work function of MWCNT yarns and sheets. ${ }^{6,10}$ For a thermionic emission cathode, the emission current density versus external electric field in the accelerating field region can be described by the Richardson-Dushman equation ${ }^{11}$

$$
J_{a}=J_{0} e^{e \sqrt{e E / 4 \pi \epsilon_{0}} / k_{B} T},
$$

where $J_{0}=A T^{2} e^{-\phi / k_{B} T}$ is the zero-field current density, $e$ is the electron charge, $\epsilon_{0}$ is the permittivity of free space, $E$ is the external electric field, $T$ is the absolute temperature, $k_{B}$ is the Boltzmann constant, $A$ is Richardson's emission constant, and $\phi$ is the work function of the cathode at absolute zero temperature. strength can be ascribed to an increase in defects in the yarn.
temperature.
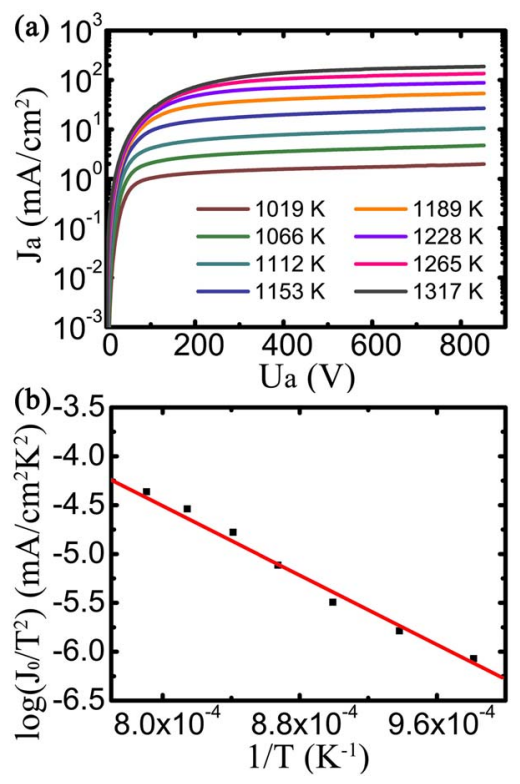

FIG. 3. (Color online) (a) $J_{a}-U_{a}$ curves for Ba-MWCNT yarn at different temperatures. (b) Experimental data (solid squares) and fitted line for a plot of $\log \left(J_{0} / T^{2}\right)$ vs $1 / T$ for Ba-MWCNT yarn.

Taking logarithms on both sides and replacing the external field $E$ by $\alpha U_{a}$, we obtain

$$
\log J_{a} \approx \log J_{0}+1.906 \frac{\sqrt{\alpha}}{T} \sqrt{U_{a}},
$$

where $U_{a}$ is the anode voltage and $\alpha$ is a constant $\left(\mathrm{cm}^{-1}\right)$ determined by the electrode geometry. If $\log J_{a}$ is plotted against $\sqrt{U_{a}}$ and fitted by a straight line, the intercept on the $y$ axis should be $\log J_{0}$.

According to the expression for zero-field current density $J_{0}$, we have

$$
\log \left(\frac{J_{0}}{T^{2}}\right)=\log A-0.4343 \frac{\phi}{k_{B} T} .
$$

A plot of $\log \left(J_{0} / T^{2}\right)$ against $1 / T$ yields a straight line with slope $-0.4343 \phi / k_{B} T$ and intercept $\log A$ [Fig. 3(b)]. The work function can thus be determined from the slope of this line. The work functions were determined to be 1.76, 2.06, and $1.73 \mathrm{eV}$ for three different samples. This variation in work function might be attributed to different surface states for different samples during measurement. ${ }^{12}$

Flexible MWCNT yarns can have tensile strength as high as $460 \mathrm{MPa}$ for a diameter of just several micrometers ${ }^{3}$ and are thus good candidates for ultrathin but strong and tough thermionic cathodes used as miniature electron sources operating at a relatively low temperature. We measured the strength of Ba-MWCNT yarns by using an Instron 5848 microtester at a strain rate of $2 \% \mathrm{~min}^{-1}$. The cross-sectional area was measured by SEM. Figure 4 shows tensile test results for MWCNT yarn after barium functionalization and further heat treatment. The tensile strength of Ba-MWCNT yarn was $\sim 420 \mathrm{MPa}$, which is similar to that reported for two-ply yarns. ${ }^{3}$ After further heating at $1200 \mathrm{~K}$ for $2 \mathrm{~h}$ under vacuum $\left(10^{-5} \mathrm{~Pa}\right)$, the tensile strength decreased to $250 \mathrm{MPa}$, which is comparable to that of pure copper. ${ }^{13}$ As the $D$ band of the Raman spectrum at $1348 \mathrm{~cm}^{-1}$ increased after heat treatment (Fig. 4, inset), the decrease in tensile 


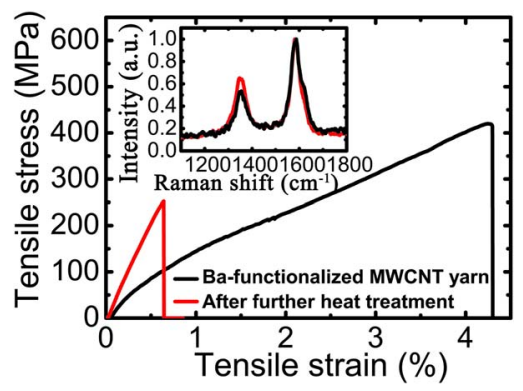

FIG. 4. (Color online) Stress-strain tests for MWCNT yarns after barium functionalization and further heat treatment. Inset: Corresponding Raman spectra.

Furthermore, since the tensile strength of pure CNT yarns decreased by $<6 \%$ after heating at $2000 \mathrm{~K}^{4}$, the increase in defects can mainly be attributed to the oxidation-reduction reaction between oxides and carbon atoms of the yarn. This drawback could be avoided by using additional activators such as $\mathrm{Mg}$ and $\mathrm{Si}$ in the oxides, as commonly applied in commercial oxide cathode production. ${ }^{14}$ The activators employed in CNT yarns should have greater activation capability than carbon to prevent CNT-oxide reaction. This should lead to higher tensile strength after heat treatment.

In summary, Ba-MWCNT yarns were easily fabricated by drawing and twisting MWCNT forests through a barium salt solution. The yarns had a work function as low as $1.73-2.06 \mathrm{eV}$ and emission current density exceeding $185 \mathrm{~mA} / \mathrm{cm}^{2}$ in a field of $850 \mathrm{~V} / 5 \mathrm{~mm}$ at $1317 \mathrm{~K}$. The tensile strength was $420 \mathrm{MPa}$ after barium functionalization and
$250 \mathrm{MPa}$ after high-temperature activation. As Ba-MWCNT yarns of micrometer diameter exhibit good thermionic and mechanical properties, they can be used as miniature thermionic electron sources in devices such as VFDs, x-ray tubes and electron guns.

The authors acknowledge financial support from the National Basic Research Program of China (2005CB623606) and NSFC (10704044 and 10572142).

${ }^{1}$ D. C. Cox, R. D. Forrest, P. R. Smith, and S. R. P. Silva, Appl. Phys. Lett. 85, 2065 (2004).

${ }^{2}$ K. L. Jiang, Q. Q. Li, and S. S. Fan, Nature (London) 419, 801 (2002).

${ }^{3}$ M. Zhang, K. R. Atkinson, and R. H. Baughman, Science 306, 1358 (2004).

${ }^{4}$ X. B. Zhang, K. L. Jiang, C. Feng, P. Liu, L. N. Zhang, J. Kong, T. H. Zhang, Q. Q. Li, and S. S. Fan, Adv. Mater. (Weinheim, Ger.) 18, 1505 (2006).

${ }^{5}$ Y. Wei, D. Weng, Y. C. Yang, X. B. Zhang, K. L. Jiang, L. Liu, and S. S. Fan, Appl. Phys. Lett. 89, 063101 (2006).

${ }^{6}$ P. Liu, Y. Wei, K. L. Jiang, Q. Sun, X. B. Zhang, and S. S. Fan, Phys. Rev. B 73, 235412 (2006).

${ }^{7}$ G. Herrmann and S. Wagener, The Oxide-Coated Cathode (Chapman and Hall, London, 1951), Vol. 1, p. 64.

${ }^{8}$ F. Jin, Y. Liu, and C. M. Day, Appl. Phys. Lett. 88, 163116 (2006).

${ }^{9}$ F. Jin, Y. Liu, C. M. Day, and S. A. Little, Carbon 45, 587 (2007).

${ }^{10}$ Y. Wei, K. L. Jiang, X. F. Feng, P. Liu, L. Liu, and S. S. Fan, Phys. Rev. B 76, 045423 (2007).

${ }^{11}$ A. L. Reimann, Thermionic Emission (Chapman and Hall, London, 1934), p. 63.

${ }^{12} \mathrm{G}$. Herrmann and S. Wagener, The Oxide-Coated Cathode (Chapman and Hall, London, 1951), Vol. 2, pp. 64-67 and 178.

${ }^{13}$ F. Cardarelli, Materials Handbook: A Concise Desktop Reference (Springer, London, 2000), p. 60.

${ }^{14}$ S. Yamamoto, Rep. Prog. Phys. 69, 181 (2006). 